\title{
Radial vibrations of an infinitely long poroelastic composite hollow circular cylinder
}

\author{
B. Shanker ${ }^{1}$, J. Manoj Kumar², S. Ahmed Shah ${ }^{3 *}$, C. Nageswara Nath ${ }^{4}$ \\ ${ }^{1}$ Department of Mathematics, Osmania University, Hyderabad, INDIA \\ ${ }^{2}$ Department of Mathematics, CMR Institute of Technology, Hyderabad, INDIA \\ $3^{3 *}$ Department of Mathematics, Deccan College of Engineering and Technology, Hyderabad, INDIA \\ ${ }^{4}$ Department of Mathematics, A.V.P.G. Centre, Hyderabad, INDIA \\ *Corresponding author,E-mail address: ahmed_shah67@yahoo.com
}

\begin{abstract}
The problem of radial vibrations of an infinitely long poroelastic composite hollow circular cylinder is solved by employing Biot's theory of wave propagation in poroelastic media. A poroelastic composite hollow cylinder consists of two concentric poroelastic cylindrical layers both of which are made of different poroelastic materials with each poroelastic material as homogeneous and isotropic. The boundaries of composite hollow poroelastic cylinder are free from stress. The frequency equations of radial vibrations of poroelastic composite hollow cylinder with rigid core, poroelastic composite solid cylinder, poroelastic composite solid cylinder with rigid casing and of rigid core and poroelastic composite bore are derived as particular cases. Non-dimensional frequency is computed as a function of ratio of thickness to inner radius of core. The results are presented graphically for two types of poroelastic composite cylinders and then discussed.
\end{abstract}

Keywords:Radial vibrations, Poroelasticity, Composite cylinder, Frequency, Rigid.

DOI: http://dx.doi.org/10.4314/ijest.v4i2.2

\section{Introduction}

McFadden (1954) studied the radial vibrations of thick-walled hollow elastic cylinders of infinite extent. Baltrukonis (1961) et al. discussed axial-shear vibrations of a composite circular cylinder. A study on radial vibrations of a poroelastic sphere was made by Paul (1974). Cui et al. (1997) and Abousleiman and Cui (1998) presented poroelastic solutions in an inclined borehole and transversely isotropic well bore cylinders. An analysis on free vibrations of multi-layered isotropic hollow spheres was made by Chen and Ding (2001). Stavsky and Greenberg (2003) studied radial vibrations of orthotropic laminated hollow spheres. Ahmed shah and Tajuddin $(2009,2011)$ discussed axial symmetric vibrations in finite composite poroelastic cylinders and torsional vibrations in thick-walled hollow poroelastic spheres. Malla Reddy and Tajuddin (2010) studied axially symmetric vibrations of composite poroelastic cylinders. Flexural wave propagation in coated poroelastic cylinders is presented by Ahmed shah (2011). Tajuddin and Ahmed shah (2010) studied the radial vibrations in thick-walled poroelastic cylinders. Tajuddin (2011) et al. discussed axial shear vibrations in poroelastic composite cylinders.

In the present analysis, radial vibrations in poroelastic composite hollow cylinder are investigated employing Biot's (1956) theory of wave propagation in porous materials. Biot's model consists of an elastic matrix permeated by a network of interconnected spaces saturated with liquid. The considered problem has wide applications in many branches of physical sciences. Fretting is essentially a contact fatigue phenomenon wherein the failure is predominantly localized near the surface. This is the case at least during the initial stages until the bulk stresses dominate and fatigue damage penetrates into the bulk material. The nature of contact between engineering components determines the near-surface state of stress, which in turn controls the severity of fretting. There are cases where the engineering components are made of materials with good mechanical properties but poor tribological properties. Fretting fatigue commonly occurs when the materials under contact are subjected to vibrations. In such 
vibrations, frequency and phase velocity are significant parameters to determine rate of energy dissipation, which in turn determines fretting fatigue. If the coating bonds strongly with the core then the coating delays crack initiation and retards crack propagation. Thus, coatings enhance the fretting fatigue strength of a component. Also when materials have good mechanical properties but poor fretting resistance, it is advantageous to provide a layer of material with good fretting resistance instead of changing entire material by means of coating. This extra layer of material can be provided by coating or by any other surfacetreatment method. The frequency equations of radial vibrations are obtained for poroelastic composite hollow cylinder and as well for some particular cases i.e., poroelastic composite hollow cylinder with rigid casing, poroelastic composite solid cylinder, poroelastic composite solid cylinder with rigid casing, poroelastic composite bore and poroelastic composite bore with rigid casing each for pervious and impervious surfaces. Non-dimensional frequency as a function of ratio of thickness to the inner radius of core is computed in each case. The results are presented graphically for two types of poroelastic composite cylinders and then discussed.

\section{Basic equations, Formulation and solution of the Problem}

The equations of motion of a homogeneous, istoropic poroelastic solid (Biot 1956) in the presence of dissipation $b$ are:

$$
\begin{aligned}
& N \nabla^{2} \mathbf{u}+(A+N) \nabla e+Q \nabla \varepsilon=\frac{\partial^{2}}{\partial t^{2}}\left(\rho_{11} \mathbf{u}+\rho_{12} \mathbf{U}\right)+b \frac{\partial}{\partial t}(\mathbf{u}-\mathbf{U}) \\
& Q \nabla e+R \nabla \varepsilon=\frac{\partial^{2}}{\partial t^{2}}\left(\rho_{12} \mathbf{u}+\rho_{22} \mathbf{U}\right)-b \frac{\partial}{\partial t}(\mathbf{u}-\mathbf{U})
\end{aligned}
$$

where $\nabla^{2}$ is the Laplacian operator $\mathbf{u}(u, v, w)$ and $\mathbf{U}(U, V, W)$ are solid and liquid displacements, respectively, while $e$ and $\varepsilon$ are dilatations of the solid and liquid respectively; $A, N, Q, R$ are poroelastic constants and $\rho_{11}, \rho_{12,} \rho_{22}$ are the mass coefficients following Biot (1956) such that the sums $\left(\rho_{11}+\rho_{12}\right)$ and $\left(\rho_{12}+\rho_{22}\right)$ are masses of solid and liquid, respectively. The poroelastic constants $A$ and $N$ correspond to familiar Lame'constants in a purely elastic solid. The coefficient $N$ represents the shear modulus of the solid. The coefficient $R$ is a measure of the pressure required on the liquid to force a certain amount of the liquid into the aggregate while the total volume remains constant. The coefficient $Q$ represents the coupling between the volume changes of solid to that of liquid.

The stresses $\sigma_{\mathrm{kl}}$ and the liquid pressure $s$ of the poroelastic solid are

$$
\begin{aligned}
& \sigma_{k l}=2 N e_{k l}+(A e+Q \varepsilon) \delta_{k l}, \quad(k, l=1,2,3) \\
& s=Q e+R \varepsilon,
\end{aligned}
$$

where $\delta_{k l}$ is the well-known Kronecker delta function.

Let $(r, \theta, \mathrm{z})$ be cylindrical polar co-ordinates. Consider a poroelastic composite hollow cylinder with two poroelastic shells bonded at the interface made of different isotropic poroelastic materials. The inner poroelastic shell is referred as core and the outer poroelastic shell is referred as casing. The prefixes $j=1,2$ are used to denote two shells related to poroelastic composite cylinder. The quantities with prefix(1) refer to the core while (2) referto the casing. The inner radius of core is ' $r_{1}$ ', outer radius of casing is ' $r$ ', whereas interface radius is ' $a$ '.

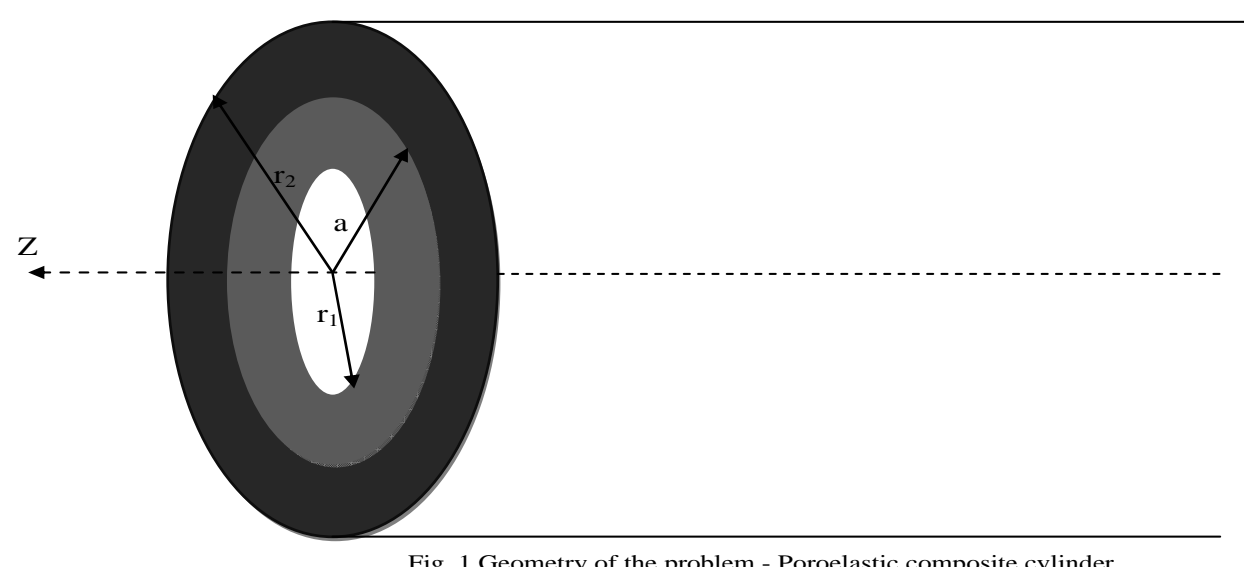

Fig. 1 Geometry of the problem - Poroelastic composite cylinder 
For radial shear vibrations, the displacement of the solid ${ }_{j} \mathbf{u}\left({ }_{j} u, 0,0\right)$ and liquid ${ }_{j} \mathbf{U}\left({ }_{j} U, 0,0\right)$ are

$$
{ }_{j} u={ }_{j} f(r) \exp (i \omega t) \text { and }{ }_{j} U={ }_{j} F(r) \exp (i \omega t)
$$

where $\omega$ is the circular frequency of wave and $t$ is the time.

Equation (1) in cylindrical polar co-ordinates when the solid and liquid displacement components ${ }_{j} u$ and ${ }_{j} U$ are independent of $\theta$ and $\mathrm{z}$ reduces to:

$$
\begin{gathered}
{ }_{j} N\left(\nabla^{2}-\frac{1}{r^{2}}\right){ }_{j} u+\left({ }_{j} A+{ }_{j} N\right) \frac{\partial e}{\partial r}+{ }_{j} Q \frac{\partial \varepsilon}{\partial r}=\frac{\partial^{2}}{\partial t^{2}}\left({ }_{j} \rho_{11} u+{ }_{j} \rho_{12} U\right)+b \frac{\partial}{\partial t}\left({ }_{j} u-{ }_{j} U\right) \\
{ }_{j} Q \frac{\partial e}{\partial r}+{ }_{j} R \frac{\partial \varepsilon}{\partial r}=\frac{\partial^{2}}{\partial t^{2}}\left({ }_{j} \rho_{12}{ }_{j} u+{ }_{j} \rho_{22} U\right)-b \frac{\partial}{\partial t}\left({ }_{j} u-{ }_{j} U\right)
\end{gathered}
$$

where e and $\varepsilon$ are the dilatations of solid and liquid respectively. The solid and liquid displacement functions can readily be evaluated from equation (4) representing plane harmonic waves, the displacements of solid and liquid ${ }_{j} u$ and ${ }_{j} U$ in the radial direction are:

$$
\begin{aligned}
& { }_{j} u=\left[{ }_{j} c_{1}{ }_{j} M_{31}(r)-{ }_{j} c_{2}{ }_{j} M_{32}(r)+{ }_{j} c_{3}{ }_{j} M_{33}(r)+{ }_{j} c_{4}{ }_{j} M_{34}(r)\right] \exp (i \omega t) \\
& { }_{j} U=-\left[{ }_{j} c_{1}{ }_{j} \delta_{1 j}^{2} M_{31}(r)+{ }_{j} c_{2}{ }_{j} \delta_{1}^{2}{ }_{j} M_{32}(r)+{ }_{j} c_{3}{ }_{j} \delta_{2}{ }^{2}{ }_{j} M_{33}(r)+{ }_{j} c_{4}{ }_{j} \delta_{2}{ }_{j}{ }_{j} M_{34}(r)\right] \exp (i \omega t)
\end{aligned}
$$

where ${ }_{j} c_{1},{ }_{j} c_{2},{ }_{j} c_{3} \&{ }_{j} c_{4}$ are the constants,

${ }_{j} M_{31}(r)=J_{1}\left({ }_{j} \xi_{1} r\right),{ }_{j} M_{32}(r)=Y_{1}\left({ }_{j} \xi_{1} r\right),{ }_{j} M_{33}(r)=J_{1}\left({ }_{j} \xi_{2} r\right), \quad{ }_{j} M_{34}(r)=Y_{1}\left({ }_{j} \xi_{2} r\right), J_{n}$ and $Y_{n}$ are Bessel functions of the $1 \mathrm{st}$ and 2nd kind of order $n$,

$$
{ }_{j} \delta_{i}^{2}=\frac{\left({ }_{j} R{ }_{j} K_{11}-{ }_{j} Q{ }_{j} K_{22}\right)-{ }_{j} V_{1}^{-2}\left({ }_{j} P{ }_{j} R-{ }_{j} Q^{2}\right)}{\left({ }_{j} R{ }_{j} K_{12}-{ }_{j} Q{ }_{j} K_{22}\right)} \text { for } i=1,2,{ }_{j} \xi_{i}=\frac{\omega}{V_{i}}(i=1,2) \text {, and }{ }_{j} P=\left({ }_{j} A+2{ }_{j} N\right) \text { for } j=1,2 \text {. }
$$

In equation (6) $V_{1}$ and $V_{2}$ are the dilatational wave velocities of $1^{\text {st }}$ and $2^{\text {nd }}$ kind, respectively, following Gardner (1962) and ${ }_{j} K_{11},{ }_{j} K_{12}$ and ${ }_{j} K_{22}$ are given by

$$
{ }_{j} K_{11}={ }_{j} \rho_{11}-\frac{i b}{\omega}, \quad{ }_{j} K_{12}={ }_{j} \rho_{12}+\frac{i b}{\omega}, \quad{ }_{j} K_{22}={ }_{j} \rho_{22}-\frac{i b}{\omega} .
$$

The dilatations of solid and liquid media following Biot (1956) are

$$
e=\left({ }_{j} f^{\prime}+\frac{1}{r}{ }_{j} f\right) \exp (i \omega t), \quad \varepsilon=\left({ }_{j} F^{\prime}+\frac{1}{r}{ }_{j} F\right) \exp (i \omega t)
$$

where a dash over a quantity denotes differentiation with respect to ' $r$ ', From equation (2), the relevant stress $\sigma_{r r}$ and the liquid pressure $s$ are

$$
\begin{aligned}
& { }_{j}\left(\sigma_{r r}+s\right)=\left[{ }_{j} c_{1} M_{11}(r)+{ }_{j} c_{2} M_{12}(r)+{ }_{j} c_{3} M_{13}(r)+{ }_{j} c_{4} M_{14}(r)\right] \exp (i \omega t) \\
& { }_{j} s=\left[{ }_{j} c_{1} M_{21}(r)+{ }_{j} c_{2} M_{22}(r)+{ }_{j} c_{3} M_{23}(r)+{ }_{j} c_{4} M_{24}(r)\right] \exp (i \omega t) \\
& { }_{j}\left(\frac{\partial s}{\partial r}\right)=\left[{ }_{j} c_{1} N_{21}(r)+{ }_{j} c_{2} N_{22}(r)+{ }_{j} c_{3} N_{23}(r)+{ }_{j} c_{4} N_{24}(r)\right] \exp (i \omega t)
\end{aligned}
$$

where $M_{i j}(r)$ and $N_{i j}(r)$ are given by 


$$
\begin{aligned}
& { }_{j} M_{11}(r)=-\frac{2}{r}\left[\left({ }_{j} Q+{ }_{j} R\right)_{j} \delta_{1}{ }^{2}-\left({ }_{j} P+{ }_{j} Q-{ }_{j} N\right)\right] J_{1}\left({ }_{j} \xi_{1} r\right)+\left[\left({ }_{j} Q+{ }_{j} R\right)_{j} \delta_{1}{ }^{2}-\left({ }_{j} P+{ }_{j} Q\right)\right]{ }_{j} \xi_{1} J_{2}\left({ }_{j} \xi_{1} r\right) \\
& { }_{j} M_{12}(r)=-\frac{2}{r}\left[\left({ }_{j} Q+{ }_{j} R\right)_{j} \delta_{1}^{2}-\left({ }_{j} P+{ }_{j} Q-{ }_{j} N\right)\right] Y_{1}\left({ }_{j} \xi_{1} r\right)+\left[\left({ }_{j} Q+{ }_{j} R\right){ }_{j} \delta_{1}{ }^{2}-\left({ }_{j} P+{ }_{j} Q\right)\right]{ }_{j} \xi_{1} Y_{2}\left({ }_{j} \xi_{1} r\right) \\
& { }_{j} M_{13}(r)=-\frac{2}{r}\left[\left({ }_{j} Q+{ }_{j} R\right){ }_{j} \delta_{2}{ }^{2}-\left({ }_{j} P+{ }_{j} Q-{ }_{j} N\right)\right] J_{1}\left({ }_{j} \xi_{1} r\right)+\left[\left({ }_{j} Q+{ }_{j} R\right){ }_{j} \delta_{2}{ }^{2}-\left({ }_{j} P+{ }_{1} Q\right)\right]{ }_{j} \xi_{2} J_{2}\left({ }_{j} \xi_{2} r\right) \\
& { }_{j} M_{14}(r)=-\frac{2}{r}\left[\left({ }_{j} Q+{ }_{j} R\right){ }_{j} \delta_{2}{ }^{2}-\left({ }_{j} P+{ }_{j} Q-{ }_{j} N\right)\right] Y_{1}\left({ }_{j} \xi_{2} r\right)+\left[\left({ }_{j} Q+{ }_{j} R\right){ }_{j} \delta_{2}{ }^{2}-\left({ }_{j} P+{ }_{j} Q\right)\right]{ }_{j} \xi_{2} Y_{2}\left({ }_{j} \xi_{2} r\right) \\
& { }_{j} M_{21}(r)=\left[-\frac{2}{r} J_{1}\left({ }_{j} \xi_{1} r\right)+{ }_{j} \xi_{1} J_{2}\left({ }_{j} \xi_{1} r\right)\right]\left[{ }_{j} R{ }_{j} \delta_{1}{ }^{2}-{ }_{j} Q\right] \\
& { }_{j} M_{22}(r)=\left[-\frac{2}{r} Y_{1}\left({ }_{j} \xi_{1} r\right)+{ }_{j} \xi_{1} Y_{2}\left({ }_{j} \xi_{1} r\right)\right]\left[{ }_{j} R{ }_{j} \delta_{1}{ }^{2}-{ }_{j} Q\right] \\
& { }_{j} M_{23}(r)=\left[-\frac{2}{r} J_{1}\left({ }_{j} \xi_{2} r\right)+{ }_{j} \xi_{2} J_{2}\left({ }_{j} \xi_{2} r\right)\right]\left[{ }_{j} R{ }_{j} \delta_{2}{ }^{2}-{ }_{j} Q\right] \\
& { }_{j} M_{24}(r)=\left[-\frac{2}{r} Y_{1}\left({ }_{j} \xi_{2} r\right)+{ }_{j} \xi_{2} Y_{2}\left({ }_{j} \xi_{2} r\right)\right]\left[{ }_{j} R{ }_{j} \delta_{2}{ }^{2}-{ }_{j} Q\right] \\
& { }_{j} N_{21}(r)={ }_{j} \xi_{1}^{2} J_{1}\left({ }_{j} \xi_{1} r\right)\left[{ }_{j} R{ }_{j} \delta_{1}^{2}-{ }_{j} Q\right] \\
& { }_{j} N_{22}(r)={ }_{j} \xi_{1}{ }^{2} Y_{1}\left({ }_{j} \xi_{1} r\right)\left[{ }_{j} R{ }_{j} \delta_{1}{ }^{2}-{ }_{j} Q\right] \\
& { }_{j} N_{23}(r)={ }_{j} \xi_{2}{ }^{2} J_{1}\left({ }_{j} \xi_{2} r\right)\left[{ }_{j} R{ }_{j} \delta_{2}{ }^{2}-{ }_{j} Q\right] \\
& { }_{j} N_{24}(r)={ }_{j} \xi_{2}{ }^{2} Y_{1}\left({ }_{j} \xi_{2} r\right)\left[{ }_{j} R{ }_{j} \delta_{2}{ }^{2}-{ }_{j} Q\right] \quad \text { for } j=1,2
\end{aligned}
$$

\section{Boundary conditions}

We assume that the outer surface of casing and inner surface of core are free from stress and there is a perfect bounding at the interface, thus the boundary conditions for stress-free vibrations of a poroelastic composite hollow cylinder in case of a pervious surface are

$$
\begin{array}{ll}
\text { at } r=r_{1} ; & { }_{1}\left(\sigma_{r r}+s\right)=0, \quad{ }_{1} s=0 \\
\text { at } r=r_{2} ; & { }_{2}\left(\sigma_{r r}+s\right)=0, \quad{ }_{2} s=0 \\
\text { at } r=a ; & { }_{2}\left(\sigma_{r r}+s\right)-{ }_{1}\left(\sigma_{r r}+s\right)=0, \quad{ }_{2} s=0={ }_{1} s, \quad{ }_{2} u-{ }_{1} u=0 .
\end{array}
$$

while the boundary conditions for stress-free vibrations of a poroelastic composite hollow cylinder in case of an impervious surface are

$$
\begin{aligned}
& \text { at } r=r_{1} ; \quad{ }_{1}\left(\sigma_{r r}+s\right)=0, \quad\left(\frac{\partial s}{\partial r}\right)=0, \\
& \text { at } r=r_{2} ; \quad{ }_{2}\left(\sigma_{r r}+s\right)=0, \quad\left(\frac{\partial s}{\partial r}\right)=0, \\
& \text { at } r=a ;{ }_{2}\left(\sigma_{r r}+s\right)-{ }_{1}\left(\sigma_{r r}+s\right)=0, \quad\left(\frac{\partial s}{\partial r}\right)={ }_{1}\left(\frac{\partial s}{\partial r}\right)=0,{ }_{2} u-{ }_{1} u=0 .
\end{aligned}
$$

Substitution of equations (9) and (10) into equation (13) results in system of eight homogeneous equations in constants ${ }_{1} C_{1},{ }_{1} C_{2},{ }_{1} C_{3},{ }_{1} C_{4},{ }_{2} C_{1},{ }_{2} C_{2},{ }_{2} C_{3},{ }_{2} C_{4}$ such a homogeneous system can have non-trivial solutions only if the determinant of the coefficients of the unknowns vanishes identically. Thus by eliminating the constants, the frequency equation of radial shear vibrations for poroelastic composite hollow cylinder for pervious surface is obtained as 


$$
\left|\begin{array}{cccccccc}
{ }_{1} M_{11}\left(r_{1}\right) & { }_{1} M_{12}\left(r_{1}\right) & { }_{1} M_{13}\left(r_{1}\right) & { }_{1} M_{14}\left(r_{1}\right) & 0 & 0 & 0 & 0 \\
{ }_{1} M_{21}\left(r_{1}\right) & { }_{1} M_{22}\left(r_{1}\right) & { }_{1} M_{23}\left(r_{1}\right) & { }_{1} M_{24}\left(r_{1}\right) & 0 & 0 & 0 & 0 \\
{ }_{1} M_{11}(a) & { }_{1} M_{12}(a) & { }_{1} M_{13}(a) & { }_{1} M_{14}(a) & { }_{2} M_{11}(a) & { }_{2} M_{12}(a) & { }_{2} M_{13}(a) & { }_{2} M_{14}(a) \\
{ }_{1} M_{21}(a) & { }_{1} M_{22}(a) & { }_{1} M_{23}(a) & { }_{1} M_{24}(a) & 0 & 0 & 0 & 0 \\
0 & 0 & 0 & 0 & { }_{2} M_{21}(a) & { }_{2} M_{22}(a) & { }_{2} M_{23}(a) & { }_{2} M_{24}(a) \\
{ }_{1} M_{31}(a) & { }_{1} M_{32}(a) & { }_{1} M_{33}(a) & { }_{1} M_{34}(a) & { }_{2} M_{31}(a) & { }_{2} M_{32}(a) & { }_{2} M_{33}(a) & { }_{2} M_{34}(a) \\
0 & 0 & 0 & 0 & { }_{2} M_{11}\left(r_{2}\right) & { }_{2} M_{12}\left(r_{2}\right) & { }_{2} M_{13}\left(r_{2}\right) & { }_{2} M_{14}\left(r_{2}\right) \\
0 & 0 & 0 & 0 & { }_{2} M_{21}\left(r_{2}\right) & { }_{2} M_{22}\left(r_{2}\right) & { }_{2} M_{23}\left(r_{2}\right) & { }_{2} M_{24}\left(r_{2}\right)
\end{array}\right|=0 \text {, }
$$

where ${ }_{j} M_{m n}(m=1,2,3 ; n=1,2,3$ and 4) are defined in equations (6) and (12).

In a similar way, substitution of equations (9) and (11) into equation (14) the frequency equation of radial shear vibrations for poroelastic composite hollow cylinder for impervious surface is obtained as

$$
\left|\begin{array}{cccccccc}
{ }_{1} N_{11}\left(r_{1}\right) & { }_{1} N_{12}\left(r_{1}\right) & { }_{1} N_{13}\left(r_{1}\right) & { }_{1} N_{14}\left(r_{1}\right) & 0 & 0 & 0 & 0 \\
{ }_{1} N_{21}\left(r_{1}\right) & { }_{1} N_{22}\left(r_{1}\right) & { }_{1} N_{23}\left(r_{1}\right) & { }_{1} N_{24}\left(r_{1}\right) & 0 & 0 & 0 & 0 \\
{ }_{1} N_{11}(a) & { }_{1} N_{12}(a) & { }_{1} N_{13}(a) & { }_{1} N_{14}(a) & { }_{2} N_{11}(a) & { }_{2} N_{12}(a) & { }_{2} N_{13}(a) & { }_{2} N_{14}(a) \\
{ }_{1} N_{21}(a) & { }_{1} N_{22}(a) & { }_{1} N_{23}(a) & { }_{1} N_{24}(a) & 0 & 0 & 0 & 0 \\
0 & 0 & 0 & 0 & { }_{2} N_{21}(a) & { }_{2} N_{22}(a) & { }_{2} N_{23}(a) & { }_{2} N_{24}(a) \\
{ }_{1} N_{31}(a) & { }_{1} N_{32}(a) & { }_{1} N_{33}(a) & { }_{1} N_{34}(a) & { }_{2} N_{31}(a) & { }_{2} N_{32}(a) & { }_{2} N_{33}(a) & { }_{2} N_{34}(a) \\
0 & 0 & 0 & 0 & { }_{2} N_{11}\left(r_{2}\right) & { }_{2} N_{12}\left(r_{2}\right) & { }_{2} N_{13}\left(r_{2}\right) & { }_{2} N_{14}\left(r_{2}\right) \\
0 & 0 & 0 & 0 & { }_{2} N_{21}\left(r_{2}\right) & { }_{2} N_{22}\left(r_{2}\right) & { }_{2} N_{23}\left(r_{2}\right) & { }_{2} N_{24}\left(r_{2}\right)
\end{array}\right|=0,
$$

where ${ }_{j} N_{m n}={ }_{j} M_{m n}$, for $m=1,3 ; n=1,2,3$ and 4

and ${ }_{j} N_{2 n}$ for $n=1,2,3,4$ are defined in eq. (12).

\section{Particular Cases}

Under suitable conditions the poroelastic composite hollow cylinder reduces to the following particular cases

4.1 Poroelastic composite hollow cylinder with rigid casing,

4.2 Poroelastic composite solid cylinder,

4.2.1 Poroelastic composite solid cylinder with rigid casing,

4.3 Poroelastic hollow cylinder,

4.3.1 Poroelastic solid cylinder,

4.4 Poroelastic composite bore,

4.4.1 Poroelastic composite bore with rigid casing,

4.4.2 Poroelastic bore.

\subsection{Poroelastic composite hollow cylinder with rigid casing}

When shear modulus of casing is very much larger than that of core, we can assume that casing is perfectly rigid. Letting the shear modulus of the casing approaches to infinity i.e., ${ }_{2} N \rightarrow \infty$, then the frequency equation (15) of radial vibrations of poroelastic composite hollow cylinder reduces to

$$
A_{1} A_{2}=0 \text {, }
$$

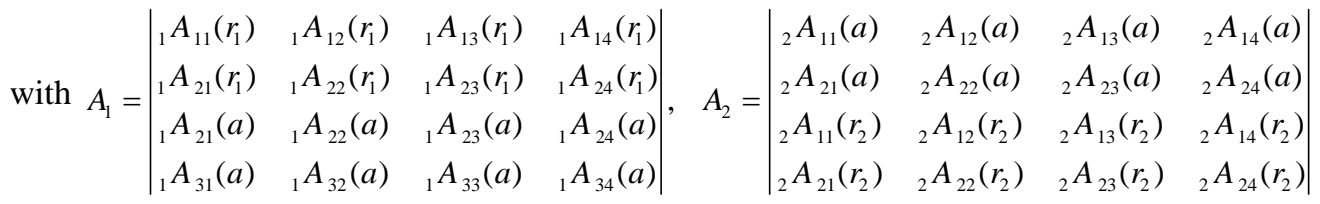

where 


$$
\begin{aligned}
& { }_{2} A_{11}(r)=-\frac{2}{r}\left[\left({ }_{2} Q+{ }_{2} R\right){ }_{2} \alpha_{1}^{2}-1\right] J_{1}\left({ }_{2} \xi_{1} r\right)+\left[\left({ }_{2} Q+{ }_{2} R\right){ }_{2} \alpha_{1}^{2}-2\right]{ }_{2} \xi_{1} J_{2}\left({ }_{2} \xi_{1} r\right) \\
& { }_{2} A_{12}(r)=-\frac{2}{r}\left[\left({ }_{2} Q+{ }_{2} R\right)_{2} \alpha \alpha_{1}^{2}-1\right] Y_{1}\left({ }_{2} \xi_{1} r\right)+\left[\left({ }_{2} Q+{ }_{2} R\right){ }_{2} \alpha_{1}^{2}-2\right]{ }_{2} \xi_{1} Y_{2}\left({ }_{2} \xi_{1} r\right) \\
& { }_{2} A_{13}(r)=-\frac{2}{r}\left[\left({ }_{2} Q+{ }_{2} R\right){ }_{2} \alpha_{2}^{2}-1\right] J_{1}\left({ }_{2} \xi_{2} r\right)+\left[\left({ }_{2} Q+{ }_{2} R\right){ }_{2} \alpha_{2}^{2}-2\right]{ }_{2} \xi_{2} J_{2}\left({ }_{2} \xi_{2} r\right) \\
& { }_{2} A_{13}(r)=-\frac{2}{r}\left[\left({ }_{2} Q+{ }_{2} R\right){ }_{2} \alpha_{2}^{2}-1\right] Y_{1}\left({ }_{2} \xi_{2} r\right)+\left[\left({ }_{2} Q+{ }_{2} R\right)_{2} \alpha_{2}^{2}-2\right]{ }_{2} \xi_{2} Y_{2}\left({ }_{2} \xi_{2} r\right) \\
& { }_{2} A_{21}(r)=\left[-\frac{2}{r} J_{1}\left({ }_{2} \xi_{1} r\right)+{ }_{2} \xi_{1} J_{2}\left({ }_{2} \xi_{1} r\right)\right]\left[{ }_{2} R{ }_{2} \alpha_{1}{ }^{2}-{ }_{2} Q\right] \\
& { }_{2} A_{22}(r)=\left[-\frac{2}{r} Y_{1}\left({ }_{2} \xi_{1} r\right)+{ }_{2} \xi_{1} Y_{2}\left({ }_{2} \xi_{1} r\right)\right]\left[{ }_{2} R{ }_{2} \alpha_{1}^{2}-{ }_{2} Q\right] \\
& { }_{2} A_{23}(r)=\left[-\frac{2}{r} J_{1}\left({ }_{2} \xi_{2} r\right)+{ }_{2} \xi_{2} J_{2}\left({ }_{2} \xi_{2} r\right)\right]\left[{ }_{2} R{ }_{2} \alpha_{2}{ }^{2}-{ }_{2} Q\right] \\
& { }_{2} A_{24}(r)=\left[-\frac{2}{r} Y_{1}\left({ }_{2} \xi_{2} r\right)+{ }_{2} \xi_{2} Y_{2}\left({ }_{2} \xi_{2} r\right)\right]\left[{ }_{2} R{ }_{2} \alpha_{2}{ }^{2}-{ }_{2} Q\right] \\
& { }_{1} A_{m n}(r)={ }_{1} M_{m n}(r), m=1,2 ; n=1,2,3,4
\end{aligned}
$$

Where ${ }_{2} \alpha_{i}^{2}=\frac{-2{ }_{2} V_{i}^{-2}{ }_{2} R}{{ }_{2} R{ }_{2} K_{12}-{ }_{2} Q{ }_{2} K_{22}}$ for $i=1,2$ and ${ }_{1} M_{m n}(m=1,2 ; n=1,2,3$ and 4$)$ are defined in equation (12).

From eq. (19) it is clear that the physical parameters in the determinants $A_{1}, A_{2}$ are, respectively, related to core and casing. Hence from eq. (18) it is clear that the vibrations of poroelastic composite hollow cylinder related to core and casing for pervious surface are uncoupled when the solid in casing is rigid, in addition we obtain $A_{1}=0$ or $A_{2}=0$. The equation

$$
A_{1}=0 \text {, }
$$

represents the frequency equation of vibrations ofporoelastic core for pervious surface when it is clamped along its outer surface, whereas the equation

$$
A_{2}=0 \text {, }
$$

represents the frequency equation of hollow rigid casing for pervious surface when the boundaries are free from stress.

In a similar way, when the solid in core is rigid, the frequency eq. (16) of vibrations of poroelastic composite hollow cylinder for an impervious surface reduces to

$$
B_{1} B_{2}=0
$$

with $B_{1}=\left|\begin{array}{llll}{ }_{1} B_{11}\left(r_{1}\right) & { }_{1} B_{12}\left(r_{1}\right) & { }_{1} B_{13}\left(r_{1}\right) & { }_{1} B_{14}\left(r_{1}\right) \\ { }_{1} B_{21}\left(r_{1}\right) & { }_{1} B_{22}\left(r_{1}\right) & { }_{1} B_{23}\left(r_{1}\right) & { }_{1} B_{24}\left(r_{1}\right) \\ { }_{1} B_{21}(a) & { }_{1} B_{22}(a) & { }_{1} B_{23}(a) & { }_{1} B_{24}(a) \\ { }_{1} B_{31}(a) & { }_{1} B_{32}(a) & { }_{1} B_{33}(a) & { }_{1} B_{34}(a)\end{array}\right|, \quad B_{2}=\left|\begin{array}{llll}{ }_{2} B_{11}(a) & { }_{2} B_{12}(a) & { }_{2} B_{13}(a) & { }_{2} B_{14}(a) \\ { }_{2} B_{21}(a) & { }_{2} B_{22}(a) & { }_{2} B_{23}(a) & { }_{2} B_{24}(a) \\ { }_{2} B_{11}\left(r_{2}\right) & { }_{2} B_{12}\left(r_{2}\right) & { }_{2} B_{13}\left(r_{2}\right) & { }_{2} B_{14}\left(r_{2}\right) \\ { }_{2} B_{21}\left(r_{2}\right) & { }_{2} B_{22}\left(r_{2}\right) & { }_{2} B_{23}\left(r_{2}\right) & { }_{2} B_{24}\left(r_{2}\right)\end{array}\right|$

where

$$
\begin{aligned}
& { }_{j} B_{m n}(r)={ }_{j} A_{m n}(r), j=1,2 ; m=1,3 ; n=1,2,3,4 \\
& { }_{1} B_{2 n}(r)={ }_{1} N_{2 n}(r), n=1,2,3,4 \\
& { }_{2} B_{21}(r)={ }_{2} \xi_{1}^{2} J_{1}\left({ }_{2} \xi_{1} r\right)\left({ }_{2} R{ }_{2} \alpha_{1}^{2}-{ }_{2} Q\right)
\end{aligned}
$$


${ }_{2} B_{22}(r)={ }_{2} \xi_{1}^{2} Y_{1}\left({ }_{2} \xi_{1} r\right)\left({ }_{2} R{ }_{2} \alpha_{1}^{2}-{ }_{2} Q\right)$

${ }_{2} B_{23}(r)={ }_{2} \xi_{2}{ }^{2} J_{1}\left({ }_{2} \xi_{2} r\right)\left({ }_{2} R{ }_{2} \alpha_{2}{ }^{2}-{ }_{2} Q\right)$

${ }_{2} B_{24}(r)={ }_{2} \xi_{2}{ }^{2} Y_{1}\left({ }_{2} \xi_{2} r\right)\left({ }_{2} R{ }_{2} \alpha_{2}{ }^{2}-{ }_{2} Q\right)$

${ }_{j} N_{2 n}$ for $n=1,2,3,4$ are defined in eq. (12) and ${ }_{j} A_{m n}$ are defined in eq. (20).

From eq. (23) it is clear that the vibrations of poroelastic composite hollow cylinder related to core and casing for impervious surface are uncoupled when the solid in casing is rigid, in addition we obtain $B_{1}=0$ or $B_{2}=0$. The equation

$$
B_{1}=0 \text {, }
$$

represents the frequency equation of vibrations ofporoelastic core for impervious surface when it is clamped along its outer surface, whereas the equation

$$
B_{2}=0 \text {, }
$$

represents the frequency equation of hollow rigid casing for impervious surface when the boundaries are free from stress.

\subsection{Poroelastic composite solid cylinder}

When the inner radius $r_{1}$ of core tends to zero, the poroelastic composite hollow cylinder reduces to poroelastic composite solid cylinder and the frequency equation (15) of vibrations of poroelastic composite hollow cylinder for pervious surface reduces to

$$
\left|\begin{array}{cccccc}
{ }_{1} M_{11}(a) & { }_{1} M_{13}(a) & { }_{2} M_{11}(a) & { }_{2} M_{12}(a) & { }_{2} M_{13}(a) & { }_{2} M_{14}(a) \\
{ }_{1} M_{21}(a) & { }_{1} M_{23}(a) & 0 & 0 & 0 & 0 \\
0 & 0 & { }_{2} M_{21}(a) & { }_{2} M_{22}(a) & { }_{2} M_{23}(a) & { }_{2} M_{24}(a) \\
{ }_{1} M_{31}(a) & { }_{1} M_{33}(a) & { }_{2} M_{31}(a) & { }_{2} M_{32}(a) & { }_{2} M_{33}(a) & { }_{2} M_{34}(a) \\
0 & 0 & { }_{2} M_{11}\left(r_{2}\right) & { }_{2} M_{12}\left(r_{2}\right) & { }_{2} M_{13}\left(r_{2}\right) & { }_{2} M_{14}\left(r_{2}\right) \\
0 & 0 & { }_{2} M_{21}\left(r_{2}\right) & { }_{2} M_{22}\left(r_{2}\right) & { }_{2} M_{23}\left(r_{2}\right) & { }_{2} M_{24}\left(r_{2}\right)
\end{array}\right|=0
$$

where the elements ${ }_{j} M_{m n}$ are defined in equations (6) and (12).

This is the frequency equation of radial vibrations of poroelastic composite solid cylinder for pervious surface.

In a similar way, using the eq. (17), the frequency equation of radial vibrations of poroelastic composite solid cylinder for an impervious surface is obtained as

$$
\left|\begin{array}{cccccc}
{ }_{1} N_{11}(a) & { }_{1} N_{13}(a) & { }_{2} N_{11}(a) & { }_{2} N_{12}(a) & { }_{2} N_{13}(a) & { }_{2} N_{14}(a) \\
{ }_{1} N_{21}(a) & { }_{1} N_{23}(a) & 0 & 0 & 0 & 0 \\
0 & 0 & { }_{2} N_{21}(a) & { }_{2} N_{22}(a) & { }_{2} N_{23}(a) & { }_{2} N_{24}(a) \\
{ }_{1} N_{31}(a) & { }_{1} N_{33}(a) & { }_{2} N_{31}(a) & { }_{2} N_{32}(a) & { }_{2} N_{33}(a) & { }_{2} N_{34}(a) \\
0 & 0 & { }_{2} N_{11}\left(r_{2}\right) & { }_{2} N_{12}\left(r_{2}\right) & { }_{2} N_{13}\left(r_{2}\right) & { }_{2} N_{14}\left(r_{2}\right) \\
0 & 0 & { }_{2} N_{21}\left(r_{2}\right) & { }_{2} N_{22}\left(r_{2}\right) & { }_{2} N_{23}\left(r_{2}\right) & { }_{2} N_{24}\left(r_{2}\right)
\end{array}\right|=0
$$

where the elements ${ }_{j} N_{m n}$ are defined in equations (12) and (17).

\subsubsection{Poroelastic composite solid cylinder with rigid casing}

When shear modulus of the casing approaches to infinity i.e., ${ }_{2} N \rightarrow \infty$, then the casing becomes perfectly rigid and the frequency equation (28) for radial vibrations of poroelastic composite solid cylinder reduces to

$$
C_{1} C_{2}=0 \text {, }
$$

with

$$
C_{1}=\left|\begin{array}{ll}
{ }_{1} C_{21}(a) & { }_{1} C_{23}(a) \\
{ }_{1} C_{31}(a) & { }_{1} C_{33}(a)
\end{array}\right|, \quad C_{2}=\left|\begin{array}{llll}
{ }_{2} C_{11}(a) & { }_{2} C_{12}(a) & { }_{2} C_{13}(a) & { }_{2} C_{14}(a) \\
{ }_{2} C_{21}(a) & { }_{2} C_{22}(a) & { }_{2} C_{23}(a) & { }_{2} C_{24}(a) \\
{ }_{2} C_{11}\left(r_{2}\right) & { }_{2} C_{12}\left(r_{2}\right) & { }_{2} C_{13}\left(r_{2}\right) & { }_{2} C_{14}\left(r_{2}\right) \\
{ }_{2} C_{21}\left(r_{2}\right) & { }_{2} C_{22}\left(r_{2}\right) & { }_{2} C_{23}\left(r_{2}\right) & { }_{2} C_{24}\left(r_{2}\right)
\end{array}\right|
$$


where ${ }_{j} C_{m n}(r)={ }_{j} A_{m n}(r), j=1,2 ; m=1,2,3 ; n=1,2,3,4$ and the elements ${ }_{j} A_{m n}(r)$ are defined in equation (20).

From eq.(28), it is clear that, when the solid in casing is rigid, the vibrations of poroelastic composite solid cylinder related to core and casing for pervious surface are uncoupled. From eq. (30) we obtain $C_{1}=0$ or $C_{2}=0$. The equation

$$
C_{1}=0 \text {, }
$$

represents the frequency equation of radial vibrations of poroelastic solid core for pervious surface when it is clamped along its outer surface, whereas the equation

$$
C_{2}=0 \text {, }
$$

represents the frequency equation of radial vibrations of hollow rigid casing for pervious surface when boundaries are free from stress.

In a similar way, when the solid in casing is rigid, the frequency eq.(29) of radial vibrations of poroelastic composite solid cylinder for impervious surface reduces to

$$
D_{1} D_{2}=0
$$

with

$$
D_{1}=\left|\begin{array}{ll}
{ }_{1} D_{21}(a) & { }_{1} D_{23}(a) \\
{ }_{1} D_{31}(a) & { }_{1} D_{33}(a)
\end{array}\right|, \quad D_{2}=\left|\begin{array}{llll}
{ }_{2} D_{11}(a) & { }_{2} D_{12}(a) & { }_{2} D_{13}(a) & { }_{2} D_{14}(a) \\
{ }_{2} D_{21}(a) & { }_{2} D_{22}(a) & { }_{2} D_{23}(a) & { }_{2} D_{24}(a) \\
{ }_{2} D_{11}\left(r_{2}\right) & { }_{2} D_{12}\left(r_{2}\right) & { }_{2} D_{13}\left(r_{2}\right) & { }_{2} D_{14}\left(r_{2}\right) \\
{ }_{2} D_{21}\left(r_{2}\right) & { }_{2} D_{22}\left(r_{2}\right) & { }_{2} D_{23}\left(r_{2}\right) & { }_{2} D_{24}\left(r_{2}\right)
\end{array}\right|
$$

where ${ }_{j} D_{m n}(r)={ }_{j} B_{m n}(r), j=1,2 ; m=1,2,3 ; n=1,2,3,4$ and the elements ${ }_{j} B_{m n}(r)$ are defined in equation (25).

From Eq. (34), it is clear that, when the solid in casing is rigid, the vibrations of poroelastic composite solid cylinder related to core and casing for impervious surface are uncoupled, also we obtain $D_{1}=0$ or $D_{2}=0$. The equation

$$
D_{1}=0 \text {, }
$$

represents the frequency equation of radial vibrations of poroelastic solid core for impervious surface when it is clamped along its outer surface, whereas the equation

$$
D_{2}=0 \text {, }
$$

represents the frequency equation of radial vibrations of hollow rigid casing for pervious surface when boundaries are free from stress.

\subsection{Poroelastic hollow cylinder}

When poroelastic constants of casing vanishes i.e. ${ }_{2} N={ }_{2} A={ }_{2} Q={ }_{2} R=0$, then poroelastic composite hollow cylinder reduces to poroelastic hollow cylinder of inner radius $r_{1}$ and outer radius $a$ and the frequency equation (15) of radial vibrations of poroelastic composite hollow cylinder reduces to

$$
\left|\begin{array}{llll}
{ }_{2} M_{11}(a) & { }_{2} M_{12}(a) & { }_{2} M_{13}(a) & { }_{2} M_{14}(a) \\
{ }_{2} M_{21}(a) & { }_{2} M_{22}(a) & { }_{2} M_{23}(a) & { }_{2} M_{24}(a) \\
{ }_{2} M_{11}\left(r_{2}\right) & { }_{2} M_{12}\left(r_{2}\right) & { }_{2} M_{13}\left(r_{2}\right) & { }_{2} M_{14}\left(r_{2}\right) \\
{ }_{2} M_{21}\left(r_{2}\right) & { }_{2} M_{22}\left(r_{2}\right) & { }_{2} M_{23}\left(r_{2}\right) & { }_{2} M_{24}\left(r_{2}\right)
\end{array}\right|=0
$$

where the elements ${ }_{j} M_{m n}$ are defined in equation (12).

Eq. (38) is the frequency equation of poroelastic hollow cylinder for pervious surface.

In a similar way, using the eq. (17), the frequency equation of radial vibrations of poroelastic hollow cylinder for an impervious surface is obtained as

$$
\left|\begin{array}{llll}
{ }_{2} N_{11}(a) & { }_{2} N_{12}(a) & { }_{2} N_{13}(a) & { }_{2} N_{14}(a) \\
{ }_{2} N_{21}(a) & { }_{2} N_{22}(a) & { }_{2} N_{23}(a) & { }_{2} N_{24}(a) \\
{ }_{2} N_{11}\left(r_{2}\right) & { }_{2} N_{12}\left(r_{2}\right) & { }_{2} N_{13}\left(r_{2}\right) & { }_{2} N_{14}\left(r_{2}\right) \\
{ }_{2} N_{21}\left(r_{2}\right) & { }_{2} N_{22}\left(r_{2}\right) & { }_{2} N_{23}\left(r_{2}\right) & { }_{2} N_{24}\left(r_{2}\right)
\end{array}\right|=0
$$

where the elements ${ }_{j} N_{m n}$ are defined in equations (12) and (17). 


\subsubsection{Poroelastic Solid cylinder}

When the inner radius $r_{1}$ of core tends to zero, the poroelastic hollow cylinder reduces to poroelastic solid cylinder and the frequency equation (38) of radial vibration of poroelastic hollow cylinder for pervious surface reduces to

$$
\left|\begin{array}{ll}
{ }_{2} M_{11}\left(r_{2}\right) & { }_{2} M_{13}\left(r_{2}\right) \\
{ }_{2} M_{21}\left(r_{2}\right) & { }_{2} M_{23}\left(r_{2}\right)
\end{array}\right|=0,
$$

where the elements ${ }_{j} M_{m n}$ are defined in equation (12).

This is the frequency equation of radial vibrations of poroelastic solid cylinder for pervious surface.

In a similar way,the frequency equation of radial vibrations of poroelastic solid cylinder for impervious surface can be obtained as

$$
\left|\begin{array}{ll}
{ }_{2} N_{11}\left(r_{2}\right) & { }_{2} N_{13}\left(r_{2}\right) \\
{ }_{2} N_{21}\left(r_{2}\right) & { }_{2} N_{23}\left(r_{2}\right)
\end{array}\right|=0,
$$

where the elements ${ }_{j} N_{m n}$ are defined in equations (12) and (17).

\subsection{Poroelastic Composite Bore}

When the outer radius $r_{2}$ of casing tends to $\infty$, the frequency equation (15) of poroelastic composite hollow cylinder for pervious surface reduces

$$
\left|\begin{array}{cccccc}
{ }_{1} M_{11}\left(r_{1}\right) & { }_{1} M_{12}\left(r_{1}\right) & { }_{1} M_{13}\left(r_{1}\right) & { }_{1} M_{14}\left(r_{1}\right) & 0 & 0 \\
{ }_{1} M_{21}\left(r_{1}\right) & { }_{1} M_{22}\left(r_{1}\right) & { }_{1} M_{23}\left(r_{1}\right) & { }_{1} M_{24}\left(r_{1}\right) & 0 & 0 \\
{ }_{1} M_{11}(a) & { }_{1} M_{12}(a) & { }_{1} M_{13}(a) & { }_{1} M_{14}(a) & { }_{2} M_{12}(a) & { }_{2} M_{14}(a) \\
{ }_{1} M_{21}(a) & { }_{1} M_{22}(a) & { }_{1} M_{23}(a) & { }_{1} M_{24}(a) & 0 & 0 \\
0 & 0 & 0 & 0 & { }_{2} M_{22}(a) & { }_{2} M_{24}(a) \\
{ }_{1} M_{31}(a) & { }_{1} M_{32}(a) & { }_{1} M_{33}(a) & { }_{1} M_{34}(a) & { }_{2} M_{32}(a) & { }_{2} M_{34}(a)
\end{array}\right|=0
$$

where the elements ${ }_{j} M_{m n}$ are defined in equation (12).

Eq. (42) is the frequency equation of radial vibrations of poroelastic composite bore for pervious surface.

Similarly, the frequency equation of radial vibrations of poroelastic composite bore for impervious surface is obtained as

$$
\left|\begin{array}{cccccc}
{ }_{1} N_{11}\left(r_{1}\right) & { }_{1} N_{12}\left(r_{1}\right) & { }_{1} N_{13}\left(r_{1}\right) & { }_{1} N_{14}\left(r_{1}\right) & 0 & 0 \\
{ }_{1} N_{21}\left(r_{1}\right) & { }_{1} N_{22}\left(r_{1}\right) & { }_{1} N_{23}\left(r_{1}\right) & { }_{1} N_{24}\left(r_{1}\right) & 0 & 0 \\
{ }_{1} N_{11}(a) & { }_{1} N_{12}(a) & { }_{1} N_{13}(a) & { }_{1} N_{14}(a) & { }_{2} N_{12}(a) & { }_{2} N_{14}(a) \\
{ }_{1} N_{21}(a) & { }_{1} N_{22}(a) & { }_{1} N_{23}(a) & { }_{1} N_{24}(a) & 0 & 0 \\
0 & 0 & 0 & 0 & { }_{2} N_{22}(a) & { }_{2} N_{24}(a) \\
{ }_{1} N_{31}(a) & { }_{1} N_{32}(a) & { }_{1} N_{33}(a) & { }_{1} N_{34}(a) & { }_{2} N_{32}(a) & { }_{2} N_{34}(a)
\end{array}\right|=0
$$

where the elements ${ }_{j} N_{m n}$ are defined in equations (12) and (17).

\subsubsection{Poroelastic Composite Bore with rigid casing}

When the solid in casing of poroelastic composite bore is rigid i.e., ${ }_{2} N \rightarrow \infty$, the frequency (42) of radial vibrations of poroelastic composite bore for pervious surface reduces to

$$
E_{1} E_{2}=0
$$


with $\quad E_{1}=\left|\begin{array}{llll}{ }_{1} E_{11}\left(r_{1}\right) & { }_{1} E_{12}\left(r_{1}\right) & { }_{1} E_{13}\left(r_{1}\right) & { }_{1} E_{14}\left(r_{1}\right) \\ { }_{1} E_{21}\left(r_{1}\right) & { }_{1} E_{22}\left(r_{1}\right) & { }_{1} E_{23}\left(r_{1}\right) & { }_{1} E_{24}\left(r_{1}\right) \\ { }_{1} E_{11}(a) & { }_{1} E_{12}(a) & { }_{1} E_{13}(a) & { }_{1} E_{14}(a) \\ { }_{1} E_{21}(a) & { }_{1} E_{22}(a) & { }_{1} E_{23}(a) & { }_{1} E_{24}(a)\end{array}\right|, \quad E_{2}=\left|\begin{array}{ll}{ }_{2} E_{22}(a) & { }_{2} E_{24}(a) \\ { }_{2} E_{32}(a) & { }_{2} E_{34}(a)\end{array}\right|$

where ${ }_{j} E_{m n}(r)={ }_{j} A_{m n}(r), j=1,2 ; m=1,2,3 ; n=1,2,3,4$ and the elements ${ }_{j} A_{m n}(r)$ are defined in equation (20).

From eq.(44), it is clear that, when the solid in casing is rigid, the vibrations of poroelastic composite bore related to core and casing for pervious surface are uncoupled. Also, we obtain $E_{1}=0$ or $E_{2}=0$. The equation

$$
E_{1}=0 \text {, }
$$

is the frequency equation of Radial vibrations of poroelastic hollow core for pervious surface when it is clamped along its outer surface, whereas the equation

$$
E_{2}=0 \text {, }
$$

represents the frequency equation of radial vibrations of poroelastic bore with rigid solid for pervious surface when boundaries are free from stress.

Similarly, the frequency (43) of radial vibrations of poroelastic composite bore for impervious surface reduces to

$$
F_{1} F_{2}=0 \text {, }
$$

with

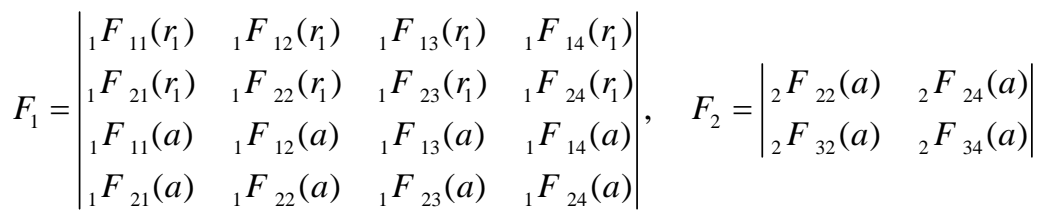

where ${ }_{j} F_{m n}(r)={ }_{j} B_{m n}(r), j=1,2 ; m=1,2,3 ; n=1,2,3,4$ and the elements ${ }_{j} B_{m n}(r)$ are defined in equation (25).

From eq.(48), it is clear that, when the solid in casing is rigid, the vibrations of poroelastic composite bore related to core and casing for impervious surface are uncoupled. Also, we obtain $F_{1}=0$ or $F_{2}=0$. The equation

$$
F_{1}=0 \text {, }
$$

is the frequency equation of radial vibrations of poroelastic hollow core for impervious surface when it is clamped along its outer surface, whereas the equation

$$
F_{2}=0 \text {, }
$$

is the frequency equation of radial vibrations of poroelastic bore with rigid solid for impervious surface when boundaries are free from stress.

\subsubsection{Poroelastic Bore}

When the poroelastic constants of core and casing of poroelastic composite bore same i.e., ${ }_{2} N={ }_{1} N,{ }_{2} A={ }_{1} A,{ }_{2} Q={ }_{1} Q$ and ${ }_{2} R={ }_{1} R$, then the poroelastic composite bore reduces to a poroelastic bore of radius $r_{1}$ and the frequency equation (42) of poroelastic composite bore reduces to

$$
\left|\begin{array}{ll}
{ }_{1} M_{12}\left(r_{1}\right) & { }_{1} M_{14}\left(r_{1}\right) \\
{ }_{1} M_{22}\left(r_{1}\right) & { }_{1} M_{24}\left(r_{1}\right)
\end{array}\right|=0
$$

where the elements ${ }_{j} M_{m n}$ are defined in equation (12).

Eq. (52) is the frequency equation of radial vibrations of poroelastic bore for pervious surface.

Similarly, the frequency equation of radial vibrations of poroelastic bore for impervious surface is obtained as

$$
\left|\begin{array}{ll}
{ }_{1} N_{12}\left(r_{1}\right) & { }_{1} N_{14}\left(r_{1}\right) \\
{ }_{1} N_{22}\left(r_{1}\right) & { }_{1} N_{24}\left(r_{1}\right)
\end{array}\right|=0,
$$

where the elements ${ }_{j} N_{m n}$ are defined in equation (17). 


\section{Non-dimensionalization of frequency equation}

The natural frequency will be real when the dissipation coefficient is zero i.e. $b=0$. For the sake of numerical work the dissipation coefficient ' $b$ ' is taken as zero and hence we obtained only real frequency. To analyze the frequency equations of radial vibrations of poroelastic composite cylinders, it is convenient to introduce the following non-dimensional parameters:

$$
\begin{aligned}
& a_{1}=\frac{{ }_{2} P}{{ }_{1} H}, \quad a_{2}=\frac{{ }_{2} Q}{{ }_{1} H}, \quad a_{3}=\frac{{ }_{2} R}{{ }_{1} H}, \quad a_{4}=\frac{{ }_{2} N}{{ }_{1} H}, d_{1}=\frac{{ }_{2} \rho_{11}}{{ }_{1} \rho}, d_{2}=\frac{{ }_{2} \rho_{12}}{{ }_{1} \rho}, d_{3}=\frac{{ }_{2} \rho_{22}}{{ }_{1} \rho} \text {, } \\
& b_{1}=\frac{{ }_{1} P}{{ }_{1} H}, \quad b_{2}=\frac{{ }_{1} Q}{{ }_{1} H}, \quad b_{3}=\frac{{ }_{1} R}{{ }_{1} H}, \quad b_{4}=\frac{{ }_{1} N}{{ }_{1} H}, \quad g_{1}=\frac{{ }_{1} \rho_{11}}{{ }_{1} \rho}, \quad g_{2}=\frac{{ }_{1} \rho_{12}}{{ }_{1} \rho}, \quad g_{3}=\frac{{ }_{1} \rho_{22}}{{ }_{1} \rho} \text {, } \\
& x_{1}=\left(\frac{1 V_{0}}{{ }_{1} V_{1}}\right)^{2}, \quad y_{1}=\left(\frac{{ }_{1} V_{0}}{{ }_{1} V_{2}}\right)^{2}, \quad x_{2}=\left(\frac{{ }_{1} V_{0}}{{ }_{2} V_{1}}\right)^{2}, y_{2}=\left(\frac{{ }_{1} V_{0}}{{ }_{2} V_{2}}\right)^{2}, \Omega=\frac{\omega h}{{ }_{1} C_{0}} \text {, }
\end{aligned}
$$

where $\Omega$ is non-dimensional frequency and

$$
{ }_{1} H={ }_{1} P+2{ }_{1} Q+{ }_{1} R, \quad{ }_{1} \rho={ }_{1} \rho_{11}+2{ }_{1} \rho_{12}+{ }_{1} \rho_{22}, \quad{ }_{1} C_{0}^{2}=\frac{{ }_{1} N}{{ }_{1} \rho}, \quad{ }_{1} V_{0}^{2}=\frac{{ }_{1} H}{{ }_{1} \rho} \text {. }
$$

Non-dimensional frequency $(\Omega)$ is calculated for two types of composite cylinders, namely composite cylinder-I and composite cylinder-II for each pervious and impervious surface.Composite cylinder-I consists of core made up of sandstone saturated with water (Yew and Jogi, 1976) and casing is made up of sandstone saturated with kerosene (Fatt, 1959), where as in composite cylinder-II, the core is sandstone saturated with kerosene and casing is sandstone saturated with water. The physical parameters of these poroelastic composite materials following equation (54) are given in Table 1.

Table - 1. Non-dimensional parameters of poroelastic composite cylinders

\begin{tabular}{|c|c|c|c|c|c|c|c|c|c|c|}
\hline $\begin{array}{c}\text { Material } \\
\text { Parameters }\end{array}$ & $\mathrm{a}_{1}$ & $\mathrm{a}_{2}$ & $\mathrm{a}_{3}$ & $\mathrm{a}_{4}$ & $\mathrm{~d}_{1}$ & $\mathrm{~d}_{2}$ & $\mathrm{~d}_{3}$ & $\mathrm{x}_{2}$ & $\mathrm{y}_{2}$ & $\mathrm{z}_{2}$ \\
\hline $\begin{array}{c}\text { Composite } \\
\text { Cylinder-I }\end{array}$ & 0.445 & 0.034 & 0.015 & 0.123 & 0.887 & -0.001 & 0.099 & 1.863 & 8.884 & 7.183 \\
\hline $\begin{array}{c}\text { Composite } \\
\text { Cylinder-II }\end{array}$ & 1.819 & 0.011 & 0.054 & 0.780 & 0.891 & 0 & 0.125 & 0.489 & 2.330 & 1.142 \\
\hline
\end{tabular}

\begin{tabular}{|c|c|c|c|c|c|c|c|c|c|}
\hline $\mathrm{b}_{1}$ & $\mathrm{~b}_{2}$ & $\mathrm{~b}_{3}$ & $\mathrm{~b}_{4}$ & $\mathrm{~g}_{1}$ & $\mathrm{~g}_{2}$ & $\mathrm{~g}_{3}$ & $\mathrm{x}_{1}$ & $\mathrm{y}_{1}$ & $\mathrm{z}_{1}$ \\
\hline 0.960 & 0.006 & 0.028 & 0.412 & 0.877 & 0 & 0.123 & 0.913 & 4.347 & 2.129 \\
\hline 0.843 & 0.065 & 0.028 & 0.234 & 0.901 & -0.001 & 0.101 & 0.999 & 4.763 & 3.851 \\
\hline
\end{tabular}

\section{Results and Discussion}

For a given poroelastic parameters, frequency equations when non-dimensionalized using equation (54), constitute a relation between non-dimensional frequency $\Omega$ and ratio of thickness of core to inner radius of core $h / r_{1}$. Different values ofr $r_{2} / a, v^{2}$., 1.1 , 1.5 and 3 are taken for numerical computation. These values of $r_{2} / a$, respectively, represent thin poroelastic casing, moderately thick poroelastic casing and thick poroelastic casing.

The frequency of radial vibrations of poroelastic composite cylinders I and II each for pervious and impervious surfaces for thin casing is presented in Fig.2. The frequency is same for pervious and impervious surfaces for both the poroelastic cylinders. The frequency of poroelastic cylinder-I is less than that of poroelastic cylinder-II for $h / r_{1} \geq 0.1$. There is sudden decrease in frequency for poroelastic cylinder-I for $\mathrm{h} / \mathrm{r}_{1} \leq 1$ after that it increasing gradually, whereas for the poroelastic cylinder-II the frequency is increasing gradually over the complete range. Fig.3 depicts the frequency of radial vibrations of poroelastic composite cylinders I 
and II each for pervious and impervious surfaces for moderately thick casing. . The frequency is same for pervious and impervious surfaces for the poroelastic cylinder-II when $\mathrm{h} / \mathrm{r}_{1} \geq 0.3$, whereas for the poroelastic cylinder-I, the frequency is same for pervious and impervious surfaces when $h / r_{1} \leq 0.3$ and $h / r_{1} \geq 0.6$. For poroelastic cylinder-I, the frequency is steadily increasing above the point $h / r_{1}=0.6$, while for the poroelastic cylinder-II it is true when $h / r_{1} \geq 0.3$. The frequency of radial vibrations of poroelastic composite cylinders I and II each for pervious and impervious surfaces for thick casing is presented in Fig.4. The variation of frequency is more in thick casing compared to thin and moderately thick casings. The frequency of radial vibrations is nearly same for pervious and impervious surfaces for poroelastic cylinder-II.

The variation of frequency of radial vibrations for poroelastic composite solid cylinder is shown in the Fig. 5 . The frequency of radial vibrations for impervious surface is less than that of pervious surface in $0.3 \leq \mathrm{h} / \mathrm{r}_{1} \leq 0.6$ in case of poroelastic cylinder-I, while in the case of poroelastic cylinder-II the frequency for impervious surface is greater than that of pervious surface when $h / r_{1}$ $\geq 0.18$. Fig. 6 shows the frequency of radial vibrations of poroelastic core when it is clamped along its outer surface. The frequency is same for pervious and impervious surfaces in case of poroelastic cylinder-I, in addition the frequency is steady when $\mathrm{h} / \mathrm{r}_{1} \leq 0.7$ and there is a sudden decrease in $0.8 \leq \mathrm{h} / \mathrm{r}_{1} \leq 0.9$. In case of poroelastic cylinder-II, the frequency is steady and the frequency for impervious surface is higher than that of pervious surface. In particular the frequency of radial vibrations forporoelastic core when it is clamped along its outer surface is linear. The variation of frequency forporoelastic casing when the solid is rigid is depicted in Fig.7. The frequency is same and steady for both the poroelastic cylinders in case of pervious surface. There is a sudden decrease in frequency for poroelastic cylinder-I incase of impervious surface when $h / r_{1} \leq 0.1$. The frequency for impervious surface is steady and higher than that of pervious surface in case of poroelastic cylinder-II. Fig. 8 depicts the variation of frequency of radial vibrations for poroelastic composite bore. It is observed that, the frequency for impervious surface is higher than that of pervious surface when $\mathrm{h} / \mathrm{r}_{1} \geq 0.27$ in case of poroelastic bore-II. In general, the frequency of poroelastic bore-I is higher than that of poroelastic bore-I.

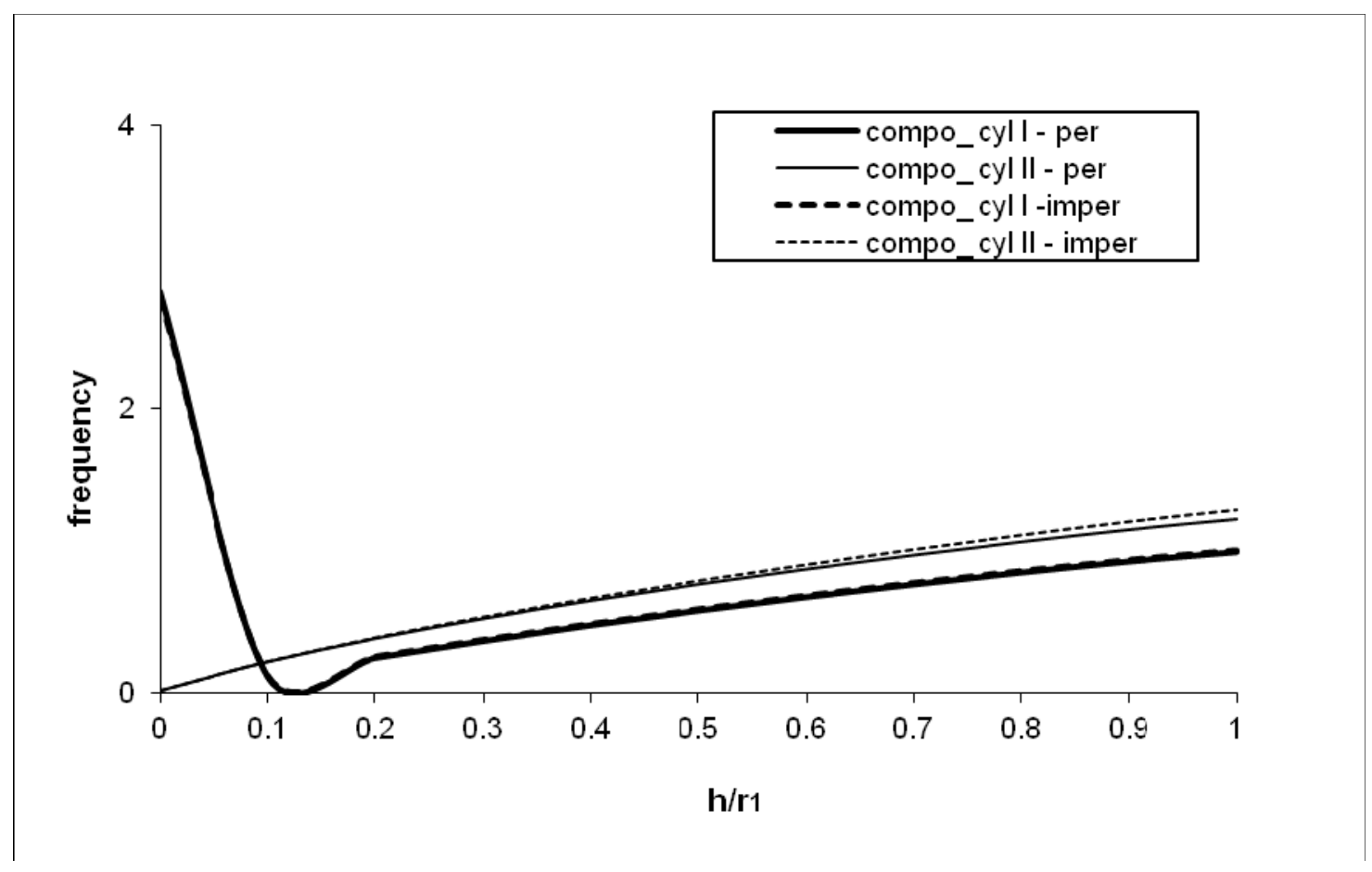

Fig.2 Variation of frequency with the ratio of thickness of core to inner radius of core for poroelastic composite hollow cylinder (Thin casing) 


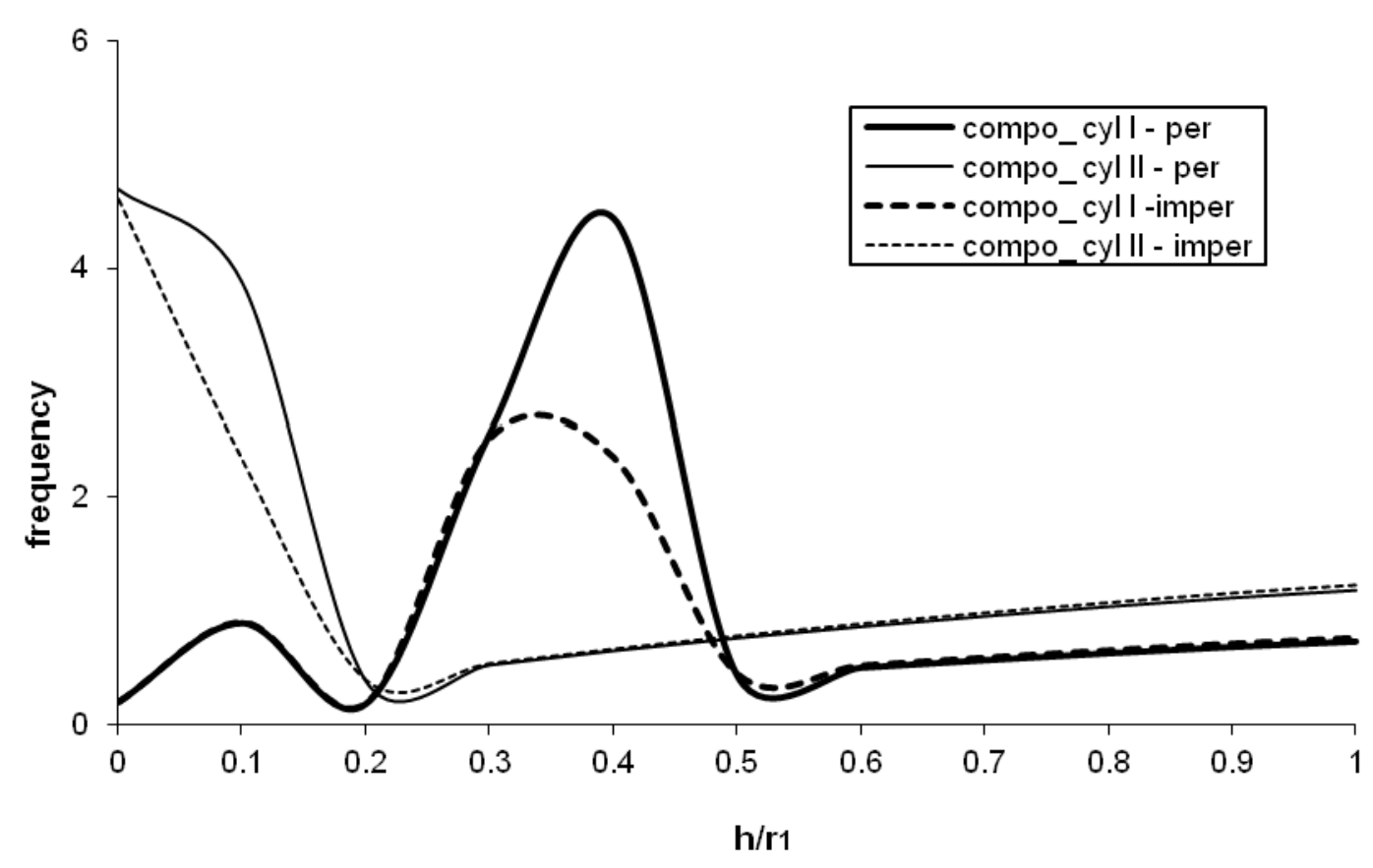

Fig.3 Variation of frequency with the ratio of thickness of core to inner radius of core for poroelastic composite hollow cylinder (Moderatedly thick casing)

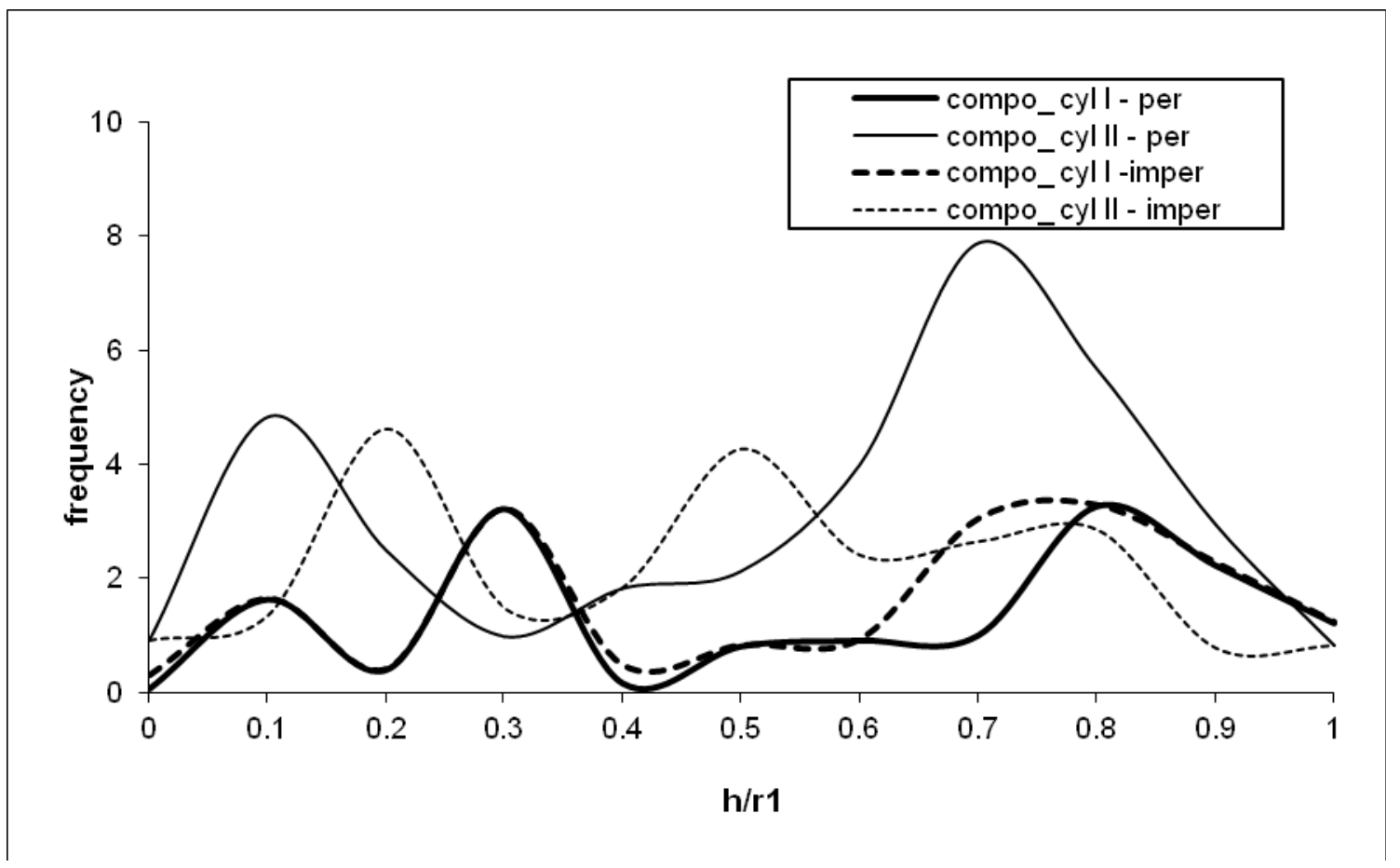

Fig.4 Variation of frequency with the ratio of thickness of core to inner radius of core for poroelastic composite hollow cylinder (Thick casing) 


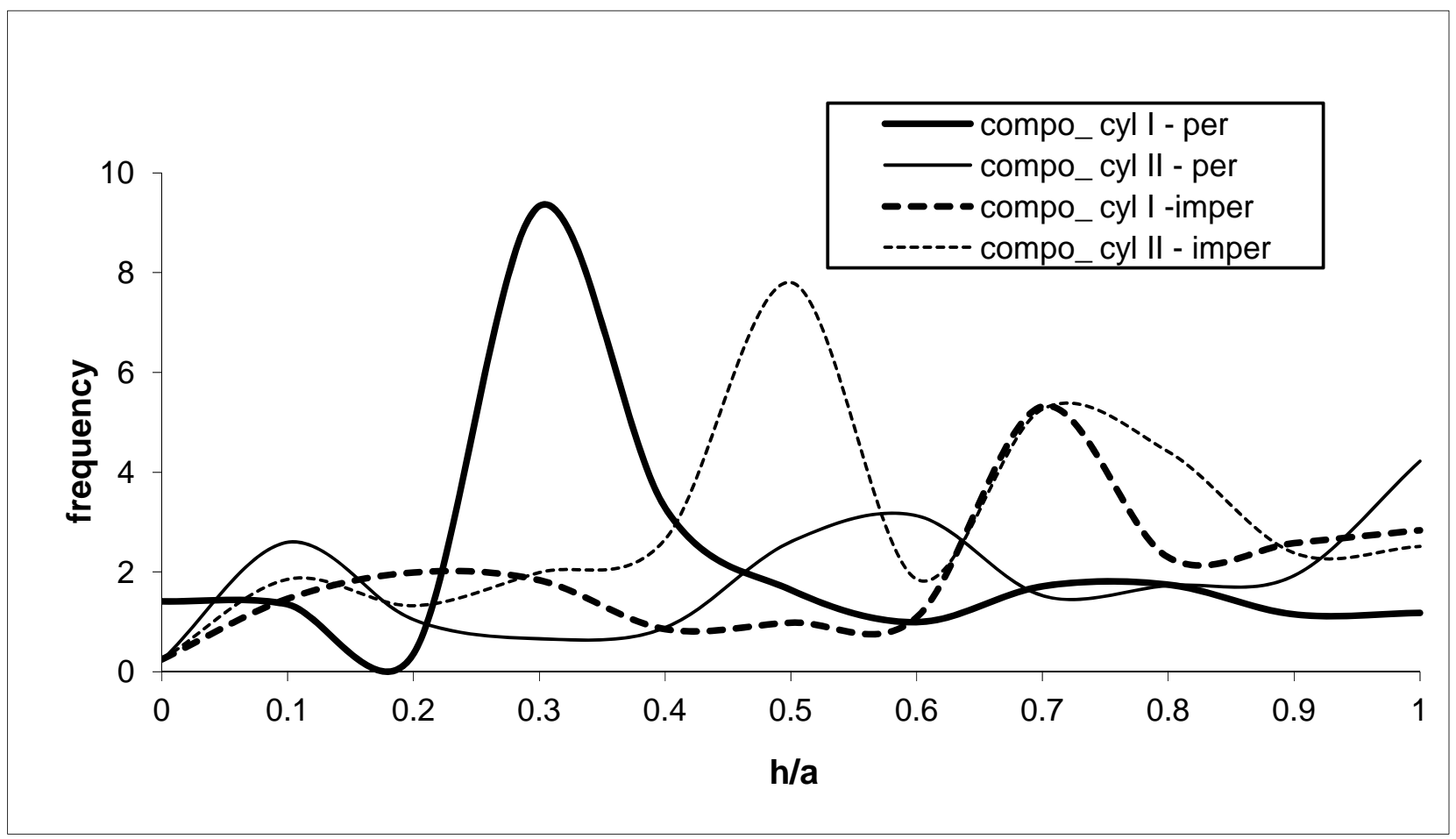

Fig.5 Variation of frequency with the ratio of thickness of casing to inner radius of casing for poroelastic composite solid cylinder

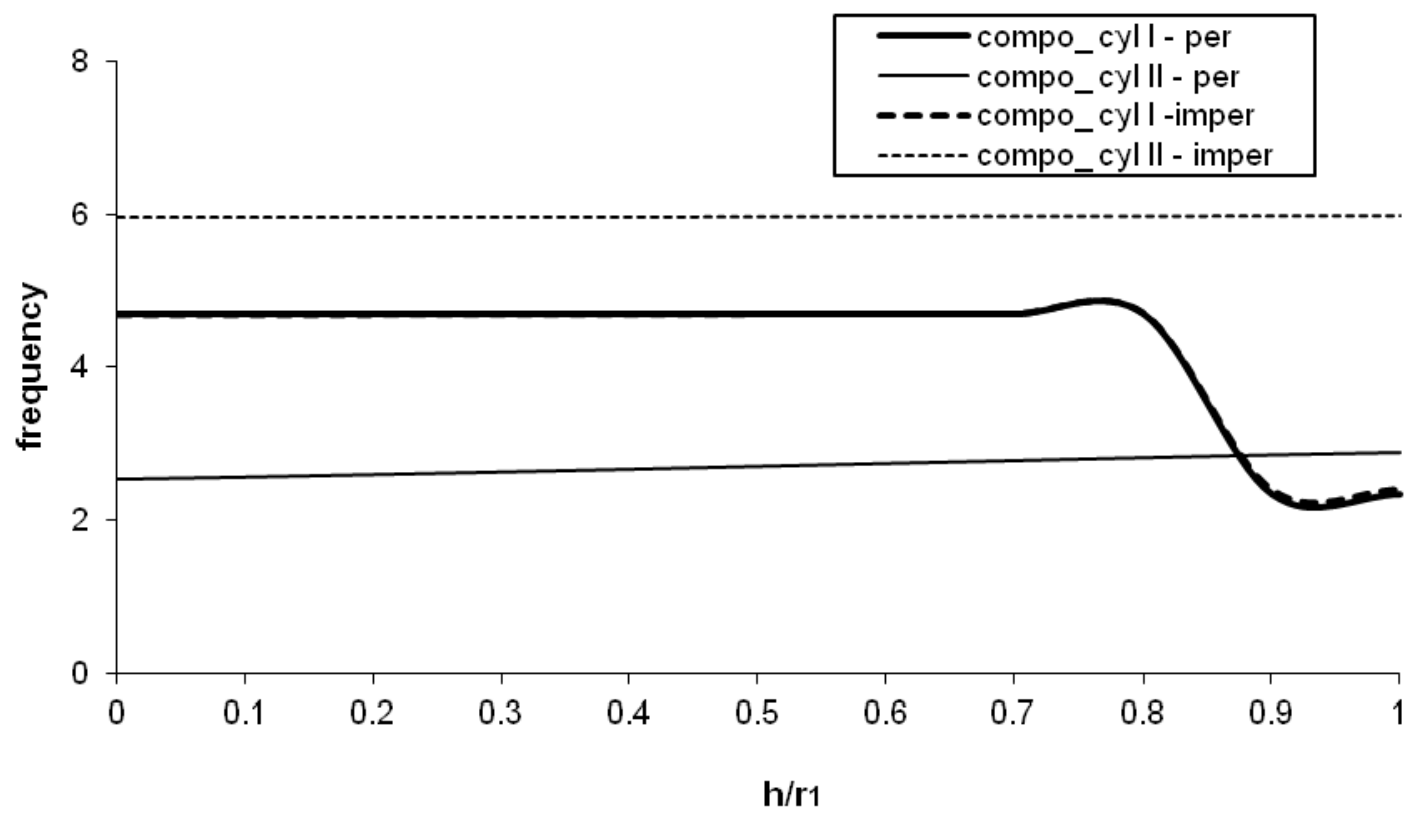

Fig.6 Variation of frequency with the ratio of thickness of core to inner radius of core for poroelastic core when it is clamped along its outer surface 


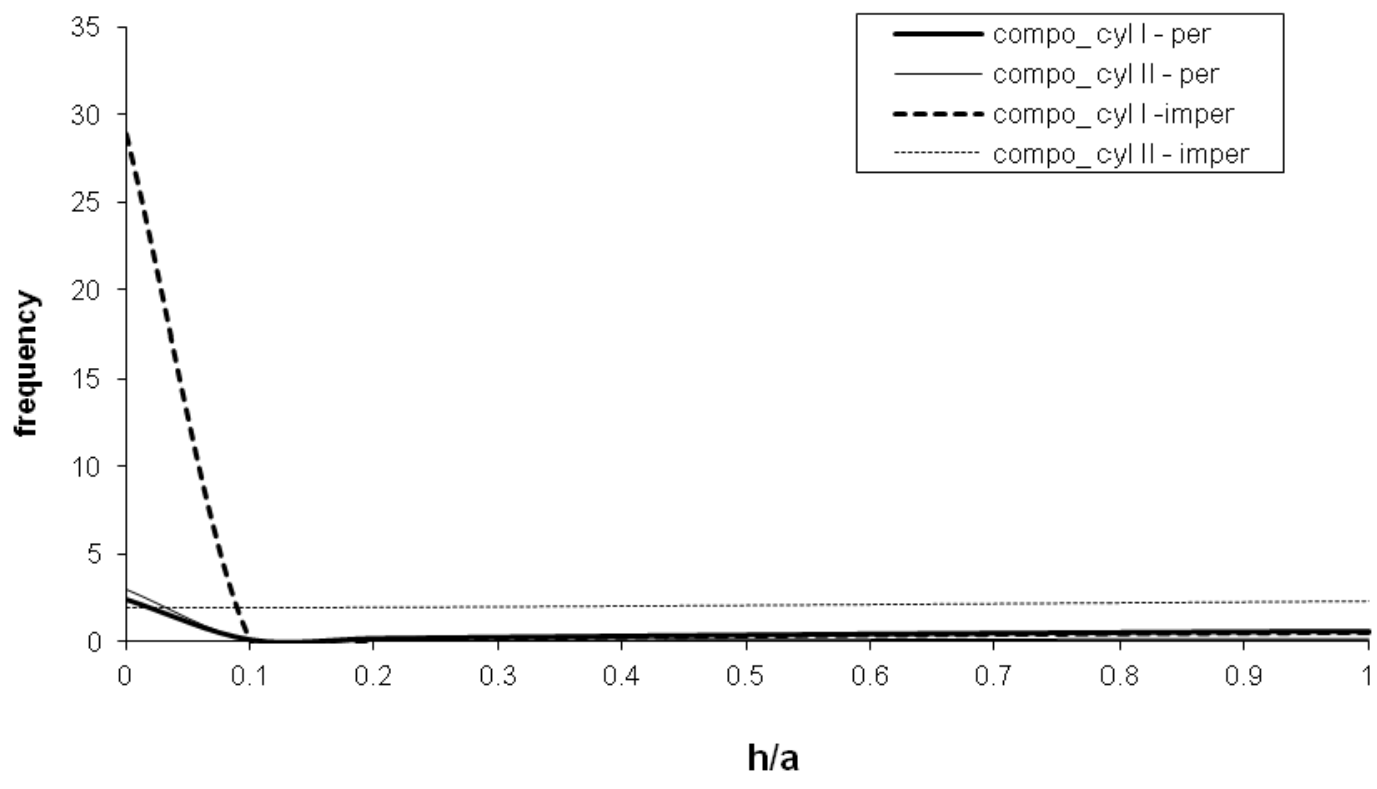

Fig.7 Variation of frequency with the ratio of thickness of casing to inner radius of casing for poroelastic casing when the solid is rigid

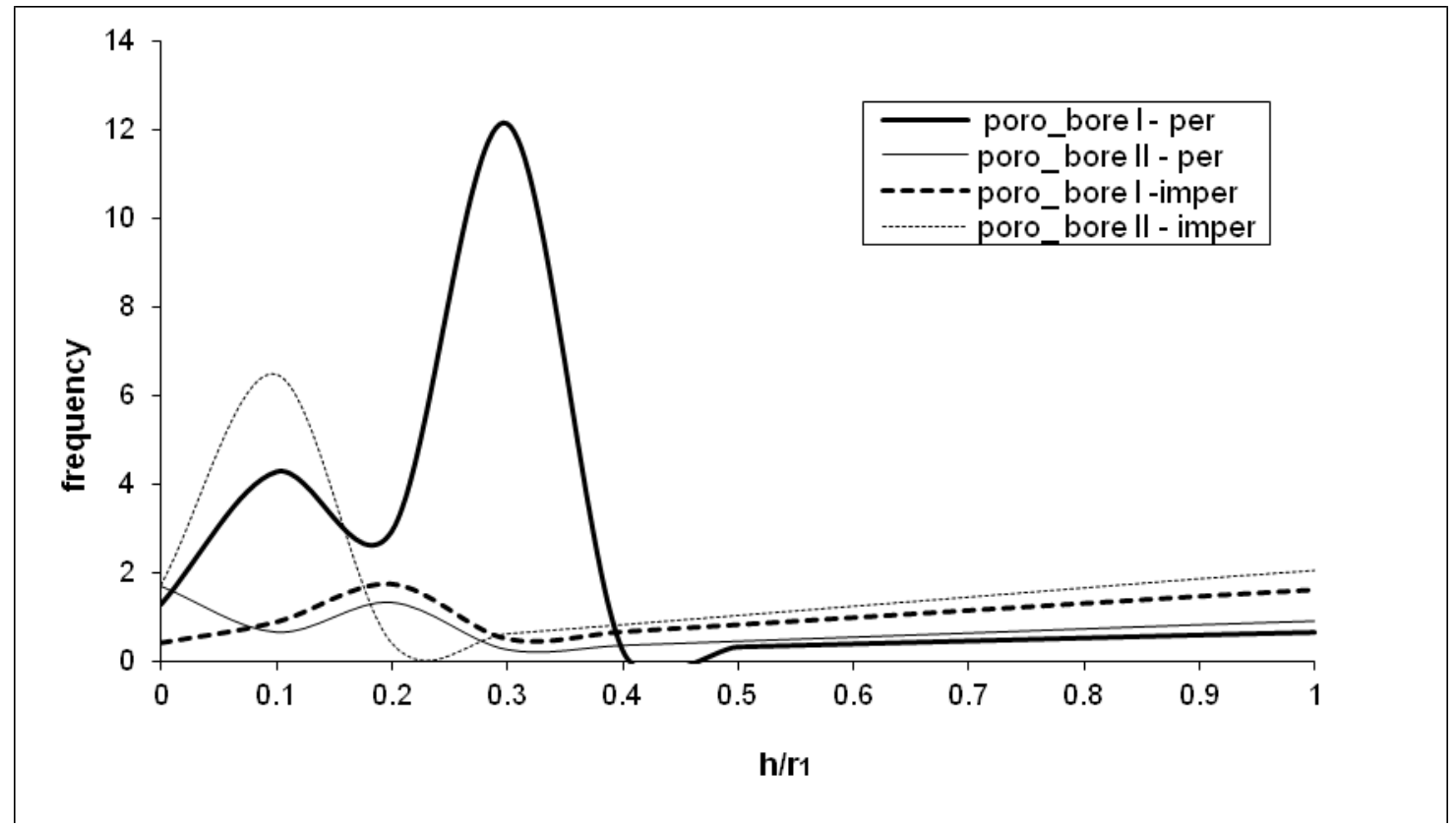

Fig.8 Variation of frequency with the ratio of thickness of core to inner radius of core for poroelastic composite bore

\section{Conclusion}

The study of radial vibrations in poroelastic composite hollow cylinders has lead to the following conclusions:

(i) The frequency of radial vibrations for poroelastic composite hollow cylinder is independent of the nature of surface in case of thin casing.

(ii) In general, the frequency of radial vibrations for poroelastic composite hollow cylinder-II is higher than that of poroelastic composite hollow cylinder-I in case of thin casing. 
(iii) The frequency of radial vibrations is steadily increasing above the point $\mathrm{h} / \mathrm{r}_{1}=0.2$ for both the poroelastic composite hollow cylinders I and II in case of thin casing..

(iv) The frequency of radial vibrations is nearly same for pervious and impervious surfaces for both the poroelastic composite cylinders I and II in case of moderately thick casing.

(v) The variation of frequency is more in case of thick casing compared to thin and moderately thick casings.

(vi) The frequency of poroelastic core when it is clamped along its outer surface is independent of nature of surface in case of poroelastic composite cylinder-I.

(vii) The frequency of poroelastic core when it is clamped along its outer surface is linear in case of poroelastic composite cylinder-II.

(viii) The frequency of poroelastic casing when the solid is rigid is linear above the point $h / \mathrm{r}_{1}=0.1$.

(ix) The variation of frequency for poroelastic bore-I is higher than that of poroelastic bore-II.

\section{Nomenclature}

$$
\begin{aligned}
& \left.{ }_{1} A,{ }_{1} N,{ }_{1} Q,{ }_{1} R\right\} \text { - Poroelastic constants } \\
& \left.{ }_{2} A,{ }_{2} N,{ }_{2} Q,{ }_{2} R\right\}- \text { Poroelastic const } \\
& \text { b - dissipation coefficient } \\
& \text { e - dilatation of solid } \\
& J_{\mathrm{n}}-\text { Bessel function of first kind of order } \mathrm{n} \\
& \mathrm{r}_{1} \text { - inner radius of core } \\
& \mathrm{r}_{2}-\text { outer radius of casing } \\
& \mathrm{s} \text { - liquid pressure } \\
& \mathrm{t} \text { - time } \\
& \text { U- liquid displacement } \\
& \mathbf{u} \text { - solid displacement }
\end{aligned}
$$

$Y_{\mathrm{n}}-$ Bessel function of second kind of order $\mathrm{n}$

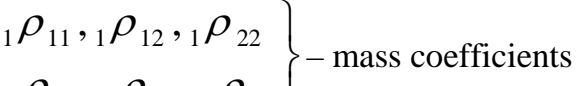

$$
\begin{aligned}
& \left.{ }_{2} \rho_{11},{ }_{2} \rho_{12},{ }_{2} \rho_{22}\right\} \\
& { }_{1} \sigma_{i j, 2} \sigma_{i j}-\text { stresses } \\
& \varepsilon \text { - dilatation of liquid } \\
& \omega \text { - circular frequency } \\
& \Omega \text { - non-dimensional frequency } \\
& \nabla^{2}-\text { Laplacian operator }
\end{aligned}
$$

\section{References}

Abousleiman, Y.N., and Cui. L., 1998. Poroelastic solutions in transversely isotropic media for wellbore cylinders, Int. J. Solids Struct., Vol. 35, pp. 4905-4929.

Ahmed shah, S., 2011. Flexural wave propagation in coated poroelastic cylinders with reference to fretting fatigue, Journal of Vibration and Control, Vol.17, pp. 1049-1064.

Ahmed shah, S., and Tajuddin, M., 2009. Axial symmetric vibrations of finite composite poroelastic cylinders, Int. J. of Applied Mechanics and Engineering, Vol.14, pp. 865-877.

Ahmed shah, S., and Tajuddin, M., 2011. Torsional vibrations of thick-walled hollow poroelastic spheres, Bull. Cal. Math. Soc., Vol. 103, pp. 161-170.

Biot, M.A., 1956. The theory of propagation of elastic waves in fluid-saturated porous solid,J. Acous. Soc. Am., Vol. 28, pp. 168178.

Baltrukonis, J.H., Gottenberg, W.G., and Schreiner, R.N., 1961. Axial-shear Vibrations of an Infinitely Long Composite Circular Cylinder, J. Acous. Soc. Am., Vol. 33, pp. 1447-1457.

Chen, W. Q., and Ding, H. J., 2001. Free vibration of multi-layered spherically isotropic hollow spheres, International Journal of Mechanical Sciences, Vol. 43, pp. 667-680. 
Cui, L., Cheng. A. H. D., and Abousleiman. Y., 1997. Poroelastic solutions of an inclined borehole, J. Appl. Mech., Vol. 64, pp. 32-38.

Fatt, I., 1959. The Biot-willis elastic coefficients for a sandstone, J. Appl. Mech., Vol. 26, pp. 296-297.

Malla Reddy, P., and Tajuddin, M., 2010. Axially symmetric vibrations of composite poroelastic cylinders in the context of fretting fatigue, Special Topics and Reviews in Porous Media, Vol. 1, pp. 311-320.

McFadden, J. A., 1954. Radial vibrations of thick-walled hollow cylinders, J. Acoust. Soc. Am., Vol. 26, pp. 714-715.

Paul, S., 1974. A note on the radial vibrations of a sphere of poroelastic material. Indian Journal of Pure and Applied Mathematics, Vol. 7, pp. 469-475.

Tajuddin, M., and Ahmed Shah, S., 2010. Radial vibrations of thick-walled hollow poroelastic cylinders, Journal of Porous Media, Vol. 13, pp. 307-318.

Tajuddin, M., Nageswara Nath, C., and Manoj Kumar, J., 2011. Axial-shear vibrations of an infinitely long poroelastic composite circular cylinder, Special Topics \& Reviews in Porous Media, Vol. 2, pp. 133-143.

Stavsky,Y., and Greenberg, J. B., 2003. Radial vibrations of orthotropic laminated hollow spheres, J. Acoust. Soc. Am., Vol. 113, pp. 847-857.

Yew, C.H., and Jogi, P.N., 1976. Study of wave motions in fluid-saturated porous rocks, J. Acoust. Soc. Am., Vol. 60, pp. 2-8.

Biographical notes

B. Shanker is with the department of Mathematics, Osmania University, Hyderabad, India

J. Manoj Kumar is working in the department of Mathematics, CMR Institute of Technology, Hyderabad, India

S. Ahmed Shah is with the department of Mathematics, Deccan college of Engineering and Technology, Hyderabad, India

C. Nageswara Nath is working in the department of Mathematics, A.V.P.G. Centre, Hyderabad, India

Received January 2012

Accepted March 2012

Final acceptance in revised form April 2012 